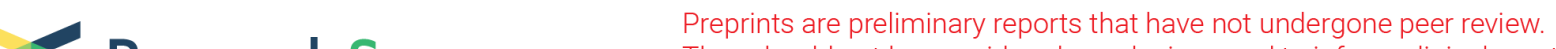 Research Square
They should not be considered conclusive, used to inform clinical practice,
or refernced by the media as validated information.
}

\section{Estimating Snakebite Risk in the Terai, Nepal: A High-Resolution Geospatial and One Health Analysis}

Carlos Ochoa ( $\sim$ carlos.ochoa@unige.ch )

University of Geneva

Marta Pittavino

University of Geneva

Sara Babo Martins

University of Geneva

Gabriel Alcoba

University Hospital of Geneva

Isabelle Bolon

University of Geneva

Rafael Ruiz de Castañeda

University of Geneva

Stéphane Joost

École Polytechnique Fédérale de Lausanne

Sanjib Sharma

B.P. Koirala Institute of Health Sciences

François Chappuis

University Hospital of Geneva

Nicolas Ray

University of Geneva

\section{Research Article}

Keywords: Snakebite risk, INLA, Nepal, GeoHealth, One Health, spatial statistics.

Posted Date: October 15th, 2021

DOI: https://doi.org/10.21203/rs.3.rs-955695/v1

License: (c) (1) This work is licensed under a Creative Commons Attribution 4.0 International License.

Read Full License 


\section{Abstract}

Most efforts to understand snakebite burden in Nepal have been localized to relatively small areas and focused on humans through epidemiological studies. We present the outcomes of a geospatial analysis of the factors influencing snakebite risk in humans and animals, based on both a national-scale multicluster random survey and, environmental, climatic, and socio-economic gridded data for the Terai region of Nepal. The resulting Integrated Nested Laplace Approximation models highlight the importance of poverty as a fundamental risk-increasing factor, augmenting the snakebite odds in humans by 63.9 times. For animals, the minimum temperature of the coldest month was the most influential covariate, increasing the snakebite odds 23.4 times. Several risk hotspots were identified along the Terai, helping to visualize at multiple administrative levels the estimated population numbers exposed to different probability risk thresholds in one year. These analyses and findings could be replicable in other countries and for other diseases.

\section{Introduction}

Currently, it is estimated that 50 people worldwide are bitten by a snake every five minutes, and one of them will die. ${ }^{1}$ From the approximate 5.4 million people bitten by snakes globally every year, up to 2.7 million are envenomed, leading to more than 400,000 disability cases and between 81,000 and 138,000 deaths. ${ }^{2-4}$ Affected populations often lack appropriate access to healthcare. ${ }^{5}$ In addition to the devastation caused to individuals, families, and communities, snakebite envenoming also causes important losses of livestock, ${ }^{6}$ which have substantial livelihood impacts for the affected households. ${ }^{7}$

In rural, agricultural populations, poverty has been extensively documented as a crucial factor linked to high snakebite incidence. Indeed, Nepal is a primarily agricultural country, with more than $65 \%$ of the population working in agriculture. ${ }^{8}$ More than $50 \%$ of its population lives in the Terai south plains ${ }^{9}$ where most of Nepal's agricultural activity is concentrated. ${ }^{10}$ This area is characterized by a high snakebite incidence both in humans and animals, recently estimated at 261 cases per 100,000 people a year, and up to 202 cases per 100,000 animals a year depending on species. ${ }^{11}$

In Nepal, as in many endemic countries, snakebite has lacked awareness and education of both the public and the medical personnel, which hinders some victims to seek appropriate medical treatment. ${ }^{10}$ Regarding domestic animals, it is even rarer for owners to reach and access veterinary services that can treat snakebite, relying more often on traditional medicine. ${ }^{12}$

Geospatial approaches have recently allowed to estimate directly and indirectly the present ${ }^{13-16}$ and future ${ }^{17}$ risk of snakebite and other spatially distributed health and social problems. ${ }^{18,19}$ However, countrywide studies using spatial epidemiology, as done in Bangladesh ${ }^{20}$ and Sri Lanka, ${ }^{21}$ still remain rare despite being invaluable to understand the burden of snakebite at national scale. Similar methods from a One Health perspective, incorporating the risks and consequences of snakebite for domestic 
animals, are almost non-existent. ${ }^{6}$ Until lately, previous studies in Nepal analysed the incidence of snakebite at district and sub-district level, either as community survey ${ }^{22}$ or based on medical records, ${ }^{23,24}$ but none addressed the incidence or risk of snakebite at a national or subnational scale. Recently, human and animal snakebite incidences were analysed nationally for the Terai, ${ }^{11,12}$ and here we analyse geospatially the risk of snakebite and the factors influencing it in humans and animals.

The World Health Organisation and the international scientific community have recognized the incompleteness of the data associated with snakebite, ${ }^{4,25}$ emphasizing the need to understand its intrinsic risk, and inform relevant stakeholders about it. It has been also emphasized that further research on ecological and environmental risk factors is necessary to develop better snakebite risk-reduction strategies, especially in places where other measures cannot be readily implemented. ${ }^{4}$ Generating this knowledge at high spatial resolution is a keystone for further actions in education, prevention, accessibility to treatment, allocation of resources, community empowerment, and health systems reinforcement in Nepal and other endemic countries.

In this study, we applied the hierarchical Bayesian, Integrated Nested Laplace Approximation (INLA) methodology to spatially analyse snakebite risk within two main types of models. In the first, we estimated the factors influencing snakebite risk for humans and domestic animals using both highly granular data from a previous multi-cluster random survey ${ }^{26}$ and geospatial gridded layers. In the second, we predicted at high spatial resolution the snakebite risk for humans based on gridded climatic and environmental data.

\section{Results}

In total, 12,998 observations (households), were available and contributed to valid data, including 154 $(1.18 \%)$ human snakebite cases, and 91 (0.7\%) domestic animal cases (all species). From the 249 clusters surveyed, 15 could not be included in the analysis due to unmatched geographic coordinates. For the full epidemiological analyses in humans and animals, see Alcoba et al. 2021, ${ }^{11}$ and Bolon et al. $2021 . .^{12}$

Modelling of snakebite risk in humans

The covariates selected for the final model of snakebite risk in humans were: food storage, straw storage, and sleeping on the floor, as well as poverty, quantified using the Poverty Probability Index (PPI), ${ }^{27}$ the average annual Normalized Difference Vegetation Index (NDVI), and distance to water. These covariates, together with the spatial random effects, were integrated in a hierarchical Bayesian logistic model (see Methods) to estimate the marginal posterior distributions of the single parameters and hyperparameters (see Table 1), and joint posterior distributions for each observed point. 
Table 1

Estimated parameters of the fitted hierarchical Bayesian models for the human and the animal risk of snakebite in the Terai. Reported statistics are the posterior marginal mean, standard deviation and mode (logit scale), as well as the corresponding mean, $90 \%$ lower- and upper-limit credible interval (odds scale). The bottom rows in each model report the spatial random effects hyperparameters. Other abbreviations: Human modification of terrestrial systems (HMTS), minimum temperature of the coldest month (BIO6), Stochastic Partial Differential Equations (SPDE).

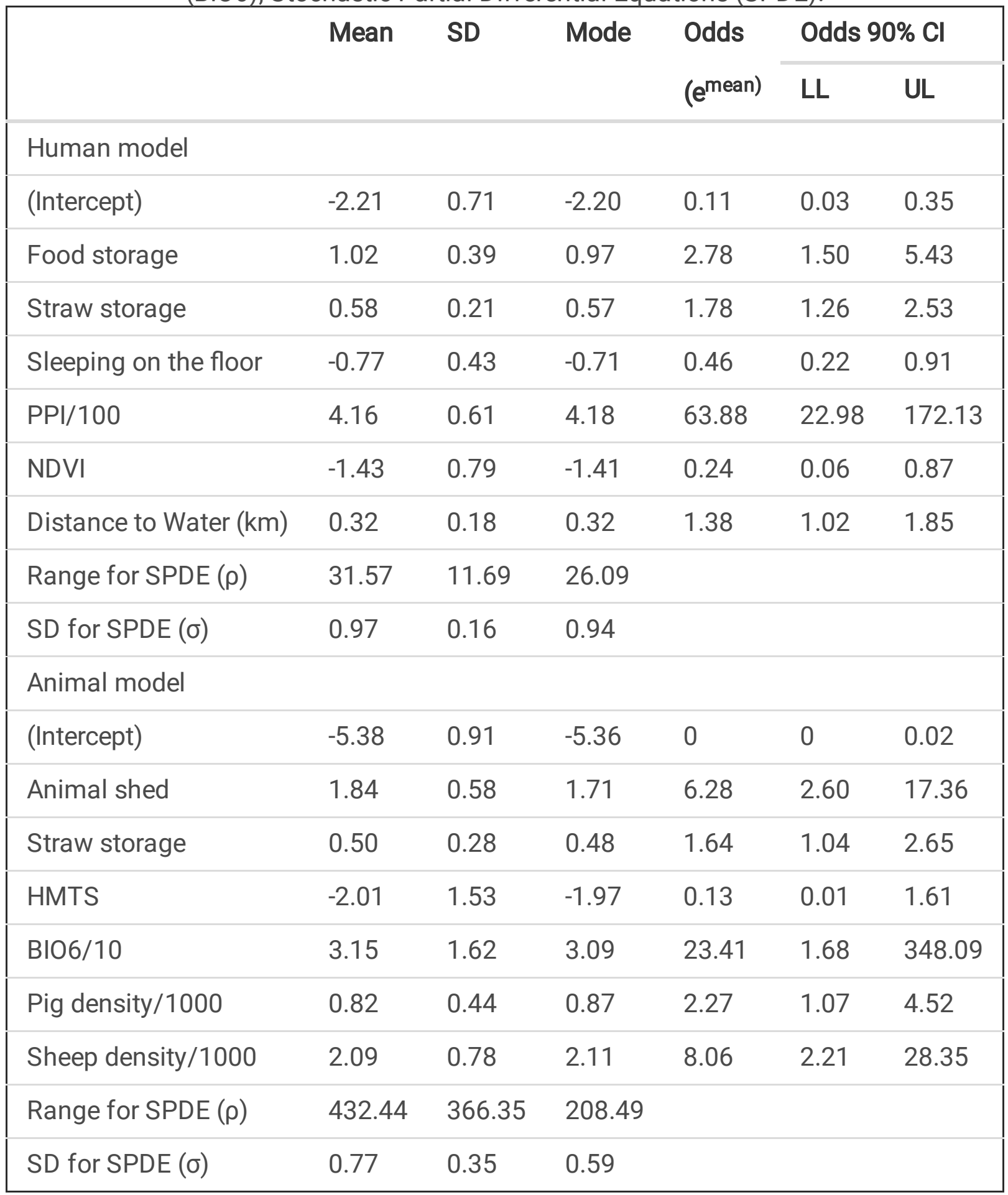

The model selection process led to a set of covariates, whose estimated coefficients fell, in all cases, into $90 \%$ credible intervals $(\mathrm{Cl})$ that did not include 1 (odds scale). Hence, they are considered significant and strongly associated with the risk of snakebite in humans. The marginal posterior distributions of all coefficients, their mean, and $90 \% \mathrm{Cl}$ are plotted in the supplementary Figure S2. 
The covariate with the strongest effect on the snakebite risk was the $P P I$, which by a change of one unit (its whole range) increased the odds of snakebite in a household 63.88 times (90\% $\mathrm{Cl} 22.98-172.13)$. Food storage was the second variable increasing the most the risk of snakebite 2.78 times $(90 \% \mathrm{Cl} 1.50-$ $5.43)$, straw storage by $78 \%(90 \% \mathrm{Cl} 1.26-2.53)$, and each additional unit $(\mathrm{km})$ in the distance to water by $38 \%(90 \% \mathrm{Cl} 1.02-1.85)$. In contrast, NDVI had the strongest 'protective' effect, with an odds ratio of 0.24 ( $90 \% \mathrm{Cl} 0.06-0.87)$, and represented a reduction of $316 \%$ to the odds of snakebite. Likewise, sleeping on the floor had a significant reducing effect of $117 \%$ in the snakebite odds $(90 \% \mathrm{Cl} 0.22-0.91)$. The estimated range $\rho$ was $31.57 \mathrm{~km}$, which represents the distance at which the spatial correlation between any two points becomes negligible. None of the relevant pairs of covariate interactions tested were statistically significant or helped explaining the changes in the response. The final model was also run without the spatial random component and the result was a clear worsening of the model fit, which verified the importance of this component.

Modelling of snakebite risk in domestic animals

The selected covariates for the final snakebite risk model in animals were: animal shed, straw storage, human modification of terrestrial systems (0-1 metric index reflecting a cumulative measure of human modification of landscapes ${ }^{28}$ ), the minimum temperature of the coldest month (WorldClim BIO6), as well as pig density and sheep density. ${ }^{29}$ The coefficients and $\mathrm{Cl}$ for parameters and hyperparameters are shown in Table 1. Here also, the interactions evaluated were neither statistically significant nor helped explaining the changes in the response variable, and therefore were not used.

The selected covariates for the final animal model were also all significant, excepting Human modification of terrestrial systems, which nevertheless had a strong 'protective' effect, equivalent to a $669 \%$ reduction of the snakebite odds $(90 \% \mathrm{Cl} 0.01-1.61)$. In contrast, all other covariates increased the odds of snakebite. The strongest effect was from $B I O 6$, where a $10^{\circ} \mathrm{C}$ temperature change represented an increase of 23.41 times the odds of snakebite ( $90 \% \mathrm{Cl} 1.68-348.09)$. Sheep density followed with an 8.06 times odd's increase for each 1000 sheep (90\% Cl 2.21-28.35), and animal shed with 6.28 times odd's increase $(90 \% \mathrm{Cl} 2.6-17.36)$. Pig density and straw storage had a less strong effect, yet they increased the odds of snakebite by 2.27 times ( $90 \% \mathrm{Cl} 1.07-4.52)$ and $64 \%(90 \% \mathrm{Cl} 1.04-2.65)$, respectively. For the animal model, the estimated range $\rho$ was $432.4 \mathrm{~km}$, showing a much larger spatial correlation between points. The marginal posterior distributions of the estimated parameters and hyperparameters, their mean, and $90 \% \mathrm{Cl}$ can be found in the supplementary Figure S3. This model was also tested without the spatial random component and the result was a distinct reduction in the model fit.

Mapping of snakebite risk and population at risk

High-resolution gridded maps $\left(1 \mathrm{~km}^{2}\right)$ of the mean snakebite-risk predicted values, their uncertainty (SD) and the estimated average number of households at risk of snakebite per year are shown in Fig. 1. The mean risk map in Fig. 1A shows several areas with increased snakebite risk along the Terai, which roughly correspond to the areas with the highest uncertainty in Fig. 1B. The map of households at risk of 
snakebite (Fig. 1C) shows two main areas with high numbers of affected population. One larger area affecting most of the districts on the eastern part of the Terai and with hotspots in three of them (Sarlahi, Saptari and Sunsari), and a smaller one in the western Terai affecting other three districts, with a hotspot in Rupandehi.

Distance to water was the covariate with the strongest, significant, risk-increasing effect (see Table 2). For each additional kilometre from a permanent water source, the odds of snakebite increased by $51 \%$ ( $90 \%$ Cl 1.12-2.04). Similar to the human risk estimation model, here also NDVI had a strong protective effect of 0.21 ( $90 \% \mathrm{Cl} 0.05-0.86$ ) equivalent to a snakebite odds-reducing effect of $476 \%$. Two more covariates were found to partially explain the changes in the response: precipitation of the driest quarter (BI017) with a snakebite odds-reducing effect of $0.12(90 \% \mathrm{Cl} 0.05-1.56)$ and the annual mean temperature (BIO1) with a protective effect of $0.28(90 \% \mathrm{Cl} 0.01-2.28)$. Despite not being significant, they had very important effects reducing the odds of snakebite, and their distributions, as seen in the supplementary Figure S4, showed that their effect was unambiguous.

Table 2

Estimated parameters of the fitted hierarchical Bayesian model for the geospatial prediction of human snakebite risk in the Terai. Reporting the posterior marginal mean, standard deviation and mode (logit scale), as well as the corresponding mean, $90 \%$ lower- and upper-limit credible intervals (odds scale). The bottom rows report the spatial random effects hyperparameters.

Other abbreviations: Stochastic Partial Differential Equations (SPDE).

\begin{tabular}{|lllllll|}
\hline & Mean & SD & Mode & Odds & \multicolumn{2}{l|}{ Odds 90\% Cl } \\
\cline { 6 - 8 } & & & & $\left(\mathbf{e}^{\text {mean }}\right)$ & LL & UL \\
\hline (Intercept) & 0.54 & 1.68 & 0.58 & 1.71 & 0.10 & 26.32 \\
\hline NDVI & -1.57 & 0.88 & -1.55 & 0.21 & 0.05 & 0.86 \\
\hline Distance Water $(k m)$ & 0.42 & 0.18 & 0.42 & 1.51 & 1.12 & 2.04 \\
\hline BI01/10 & -1.29 & 1.04 & -1.36 & 0.28 & 0.01 & 2.28 \\
\hline BIO17/100 & -2.12 & 1.78 & -2.19 & 0.12 & 0.05 & 1.56 \\
\hline Range for SPDE $(\rho)$ & 28.25 & 10.58 & 23.25 & & & \\
\hline SD for SPDE $(\sigma)$ & 0.99 & 0.15 & 0.97 & & & \\
\hline
\end{tabular}

Based on the map of households at risk and the average number of people per household in the Terai $(5.27)^{9}$ we extracted summary statistics for the districts' adjusted population (i.e., not considering highly populated Village Development Committees - VDCs) living in areas at three different risk thresholds ( $\geq 0.01, \geq 0.05$ and $\geq 0.1$, see Table 3 ). Choropleth maps aggregating the estimated population exposed at snakebite risks $\geq 0.01$ and $\geq 0.05$ per VDC during 12 months are shown in Fig. 2. Additionally, equivalent maps for municipality and district are displayed in the supplementary Figures S5 and S6. Enlargeable, 
interactive versions of these maps can be found in the supplementary HTML files S1-S6. No comparable maps were plotted for the $\geq 0.1$ risk, since only very few administrative units had any population over that risk threshold. We found three main predicted hotspots in the districts of Saptari, Makwanpur, and Sarlahi, where populations with at least 692 people are expected to be at an elevated snakebite risk $\geq 0.1$ (see Table 3). When the risk threshold is set to a lower value $(\geq 0.01)$, larger numbers of people in many more districts fall into that risk category, e.g., going up to 613,043 people in Rupandehi. This could represent around 6,130 possible snakebite victims in that district in one year. In addition to Rupandehi (91.41), six more districts in the $\geq 0.01$ risk class have percentages of adjusted 'rural' population at risk higher than 70\%, namely Saptari (86.16), Mahottari (86.11), Dhanusa (80.46), Makwanpur (73.89), Siraha (72.17), and Dang (70.79). 
Table 3

Estimated adjusted population for 2018 in each district of the Terai (WorldPop), and population living in areas with snakebite risks larger or equal to $0.01,0.05$, or 0.1 . All, the adjusted district population and the risk classes exclude the highly populated urban VDCs, removed by design, where no estimation was done.

\begin{tabular}{|c|c|c|c|c|c|}
\hline Region & District & $\begin{array}{l}\text { Adjusted } \\
\text { pop. (2018) }\end{array}$ & $\begin{array}{l}\text { Population at } \\
\geq 0.01 \text { risk, (\%) }\end{array}$ & $\begin{array}{l}\text { Population at } \\
\geq 0.05 \text { risk, }(\%)\end{array}$ & $\begin{array}{l}\text { Population at } \\
\geq 0.1 \text { risk, (\%) }\end{array}$ \\
\hline East & Jhapa & 369,014 & $225,609(61.14)$ & 0 & 0 \\
\hline East & Morang & 499,966 & $227,215(45.45)$ & $24(0.00)$ & 0 \\
\hline East & Saptari & 509,774 & $439,229(86.16)$ & 87,351 (17.14) & $1257(0.25)$ \\
\hline East & Siraha & 789,571 & $569,841(72.17)$ & 0 & 0 \\
\hline East & Sunsari & $1,327,568$ & $511,269(38.51)$ & 0 & 0 \\
\hline East & Udayapur & 249,598 & $151,315(60.62)$ & $569(0.23)$ & 0 \\
\hline Central & Bara & 541,445 & 9504 (1.76) & 0 & 0 \\
\hline Central & Chitawan & 329,361 & 7008 (2.13) & 0 & 0 \\
\hline Central & Dhanusa & 634,001 & $510,105(80.46)$ & 0 & 0 \\
\hline Central & Mahottari & 512,858 & $441,618(86.11)$ & 109 (0.02) & 0 \\
\hline Central & Makwanpur & 256,102 & 189,236 (73.89) & 8002 (3.12) & $1025(0.40)$ \\
\hline Central & Parsa & $1,161,894$ & $140(0.01)$ & 0 & 0 \\
\hline Central & Rautahat & 575,095 & $2136(0.37)$ & 0 & 0 \\
\hline Central & Sarlahi & 646,882 & $426,967(66.00)$ & 24,137 (3.73) & $692(0.11)$ \\
\hline West & Kapilbastu & 581,841 & $256,280(44.05)$ & $1948(0.33)$ & 0 \\
\hline West & Nawalparasi & 442,365 & $223,614(50.55)$ & $463(0.10)$ & 0 \\
\hline West & Rupandehi & 670,644 & $613,043(91.41)$ & 9717 (1.45) & 0 \\
\hline Mid-Western & Banke & 330,191 & $125,581(38.03)$ & $811(0.25)$ & 0 \\
\hline Mid-Western & Bardiya & 277,439 & 31,578 (11.38) & $124(0.04)$ & 0 \\
\hline Mid-Western & Dang & 396,505 & $280,690(70.79)$ & $1490(0.38)$ & $36(0.01)$ \\
\hline Mid-Western & Surkhet & 157,496 & 52,701 (33.46) & 0 & 0 \\
\hline Far-Western & Kailali & 669,770 & 7931 (1.18) & 0 & 0 \\
\hline Far-Western & Kanchanpur & 728,439 & $22,669(3.11)$ & 0 & 0 \\
\hline
\end{tabular}

\section{Discussion}


Our results showed that covariates at different geographical scales (national and local) may have important effects on the risk of snakebite, both for humans and animals. The results indicate that the risk of snakebite in the Terai varies at national scale between clusters and at local scale between households. The evaluation of the final models without spatial random components and the worsening of the models' goodness of fit as a result highlighted how snakebite risk and its determining factors are indeed, spatially structured.

A strong association between high snakebite incidence and mortality, and poverty was established from the analysis of 138 countries affected by the disease. ${ }^{30}$ In this study, we identified the $P P I$, an indicator for poverty, as a highly influential risk-increasing factor for humans. This not only confirms the critical role of poverty as a driver for this Neglected Tropical Disease, but also offers the possibility to use a standardized index at individual household scale for similar studies. Chaves et al. ${ }^{31}$ used the Poverty Gap, which is a simpler index expressing how far a person is from the average national poverty line, but to our knowledge, no study has used PPI for snakebite in any way. Applying PPI as a snakebite risk predictor also addresses previous experts calls on an Ecohealth approach to consider the relationship between the structural characteristics of houses, poverty, and snakebite. ${ }^{32}$

Three of the survey covariates had significant effects on the odds of snakebite. Food storage and straw storage increased them, while sleeping on the floor reduce them. The effect of the first two covariates is likely to be related to prey availability, represented by rodents, which are attracted by food and shelter sources. Both food and straw are very often stored near dwellings, which in the end multiply the number of possible encounters with humans and domestic animals, and the hunting snakes. ${ }^{20}$ The expected snakebite risk reduction effect by sleeping on the floor is unclear though, since on the one hand, the opposite was previously reported (at least for rural Hindus), ${ }^{33,34}$ while on the other hand, a study reported no significant difference in snakebite cases between sleeping on a cot or on the floor. ${ }^{35}$ Thus, this might be a confounding result, since this covariate is already bounded to a specific daytime and activity, and has been mainly linked to the risk of being bitten by Kraits (Bungarus spp.) hunting at night inside houses. $^{22}$

For both the general human risk model and its equivalent prediction model, the covariate Distance to water had a significant risk-increasing effect. For each additional $\mathrm{km}$ in distance from permanent water sources, the odds of snakebite increased by $38 \%$ and $51 \%$, respectively. There are two important aspects to consider in relation to water sources. One is, as in this study, the distance from large, constant water sources, which usually represents stable environments subjected to less hydric stress. The second (not considered here) are the human-made water sources, such as ponds, reservoirs, and paddy fields that change often, are usually closer to human dwellings, and are known to attract some medically important venomous snakes (MIVS). ${ }^{5}$ Studies on snake migration and home range use have concluded that depending on species and ecological conditions, snakes can move between a few tens of meters per day and more than $10 \mathrm{~km}$ between seasons, while searching for water and prey resources. ${ }^{36}$ In sub-tropical regions like the Terai, snakes living closer to continuous sources of water and vegetation should have 
easier access to a wider variety of prey. On the contrary, those living in agricultural areas might need to scout farther in the search for resources, encountering human-made waterbodies and prey, such as rodents ${ }^{37}$ and amphibians, abundant in this region. ${ }^{10}$ Further studies considering all sources of water and possibly species richness would be necessary to completely understand the effect of this covariate.

Another important factor was the NDVI, which is a commonly used value to express photosynthetic activity, leaf production and in summary the 'greenness' of the environment. ${ }^{38}$ As for other covariates, its interpretation depends on the study circumstances. In Iran, it was considered an indicator of prey availability for snakes and linked to snake habitat suitability. ${ }^{14}$ Elevated $N D V I$ values have been associated with higher number of hospitalizations in Nigeria and northern Ghana, in particular during the periods of high agricultural activity, which is also related to higher snake-human contact and higher snakebite incidence. ${ }^{38}$ In our study, its 'protective' effect can indeed be the consequence of better access to prey associated with healthier ecosystems, explained in the Terai by the higher $N D V I$ values of the multiple dense forests distributed along the region. In addition, the averaged NDVIvalues for agricultural areas should be lower than those for perennial forests, because they include the highs and lows of production and harvest.

Environmental drivers like temperature and precipitation are common factors in geospatial analyses of snakebite. ${ }^{13,14,17,39}$ They are found in many cases to be the main factors modulating the incidence or risk of snakebite, while varying in importance according to study conditions. For example, in Iran, precipitation seasonality was the most prevalent climatic covariate determining the habitat suitability leading to snakebite risk, ${ }^{14}$ while in Mozambique, temperature seasonality was the predominant covariate. ${ }^{13}$ Despite the Terai's sub-tropical climate, the range of the average minimum temperature of the coldest month (BIO6) was $1.8-10.9^{\circ} \mathrm{C}$. For our snakebite risk analysis in animals, an increase of $10^{\circ} \mathrm{C}$ of $\mathrm{BIO} 6$ between any two points represented an increase in the odds of snakebite of 23.41 times. For snakes, this range could be the difference between total lethargy and partial activity, ${ }^{40}$ which could lead to increased numbers of snakebites. In addition, and according to the production and holding practices of domestic animals in the Terai, this temperature range can also represent the difference between animals (mainly ruminants) being kept in sheds when in the lower range limits, or being let out of them in the upper limits, which would again increase the chances of encounters with snakes.

Similarly, for the animal model, pig density and sheep density, significantly influenced the variation in the risk of snakebite for animals in the Terai. This could be due to the conditions in which the animals and their feed are kept, favouring environments that are beneficial for either snakes or their preys. At more local scales, more than the distribution, the presence of other animal species could be instead the factor associated with higher snakebite rates. ${ }^{12}$ However, since the available data on domestic animal density was produced more than 10 years ago, and the animal population has grown substantially in the last years in Nepal, this outcome should be interpreted with caution.

For the animal risk, the possession of an animal shed also significantly increased the odds of snakebite. Similar to straw storage, animal sheds and similar constructions offer some shelter and the same time 
attract small (prey) animals, both of which are likely to attract snakes, increasing snakebite risk for the animals using the shed. If in addition, the sheds function as coops, the snake hunting behaviour might be instead targeted to chicks and chickens. ${ }^{12}$ This effect might be inverted when the number of animals kept increases substantially and the resources invested in the pen or coop makes it more resilient to snakes' incursions.

The human modification of terrestrial systems was the only non-significant covariate in the animal risk model. However, its strong, risk-reducing effect still seems to explain a lot of the response variation, thus, it was kept. Its change in one unit, i.e., going from pristine to fully modified environment, decreased the odds of snakebite by 0.13 (equivalent to a $669 \%$ reduction), which agrees with previous national survey results from Sri Lanka. ${ }^{21}$

For our human risk prediction model, only four covariates were either significant or helped explaining the changes in the response, which might represent a lower explanatory power. However, extensive model selection did not lead to any other covariate that could explain the variation in the response. In addition to the Distance to water and NDVI, two climatic covariates explained some of that variation. They were the precipitation of the driest quarter (BI017) and the mean annual temperature (BI01). For $\mathrm{BIO17}$, an increase of $100 \mathrm{~mm}$ of rain during the driest months of the year represented a risk-reduction effect equivalent to $733 \%$. This agrees with the results of the Distance to water, suggesting that the additional availability of resources during water shortage periods, i.e., almost four times more rain (BIO17 range: 18$71 \mathrm{~mm}$ ), could locally improve ecological conditions for snakes also leading to less scouting and less human encounters. Multiple studies have analysed the multilevel ecological effects of droughts, e.g., reducing snakes' prey and leading snakes to engage in riskier behaviours. ${ }^{41,42}$ For $\mathrm{BIO} 1$, the protective effect was weaker. An increase of $10^{\circ} \mathrm{C}$ represented a reduction of the odds of snakebite equivalent to $257 \%$. Average temperatures for specific locations are difficult to interpret, since they might depend on mild highs and lows, strong highs and lows, or relative combinations of both. Thus, despite having a relatively important effect on the response, this effect still might be the consequence of confounding and unknown interactions.

Several other evaluated covariates, for both humans and animals, showed a negligible effect on describing the response, were not significant while having very large uncertainties, or both. Consequently, they were discarded as predicting factors. For the list of baseline covariates evaluated, see supplementary Table S1. For a complete list of available survey covariates, see Alcoba et al. 2021. ${ }^{26}$

Some of our discarded covariates have been important in other studies, for example, to quantify snakebite risk based on reclassification methods of covariates such as habitat suitability, species presence, or envenoming severity. ${ }^{13,14,17,39,43}$ These methods are especially relevant when one species (or very few) is the cause of most snakebite cases, and has differentiated optimal and sub-optimal habitats. In Nepal, and particularly in the Terai, there are at least two, and sometimes more than 10 MIVS with overlapping distributions. ${ }^{44}$ Thus, it could be said that practically the whole region offers suitable habitat for multiple MIVS. In our analysis, species richness was removed, as it showed almost no effect on the 
response. A recent meta-analysis reported an equivalent result at global scale, finding no significant difference between the number of venomous snake species in tropical and temperate locations, while the number of snakebites is clearly higher in tropical areas. ${ }^{45}$ These results suggested that high incidence of snakebite is unrelated to species richness, but instead related to other factors like the number of people working in agricultural environments. ${ }^{21,30,45}$ Another important driver of snakebite incidence has been population density. ${ }^{45}$ In our study, however, any possible effect from population density on the risk was diminished by the random selection of households at specific numbers during study design. This was later confirmed by the minimal effect of population density as covariate in the human risk analysis.

This study presented a few limitations. For instance, despite the capacity of the INLA method to borrow strength from neighbouring observations and areas, the selection of adequate covariates with enough explanatory power still depends greatly on the number of snakebite cases, which even for a national scale study like this remains small. Also, some of the covariates with the strongest explanatory power came from our household survey, which prevented their use for generalized spatial prediction models. Concerning the animal risk analysis, due to the small number of snakebite cases we opted to aggregate all animal species and consider a grouped response. Thus, for a spatial analysis of animal risk, it was not worth it to consider each species, since that would dilute further an already sparse dataset in individual models and selection processes. Moreover, the data gathered for animals was dependent on the random selection of (human) households and unrelated to the current distribution of animal populations. This, in addition to the possible number of dry bites that go unnoticed, might be responsible for the low number of animal victims recorded (even combined across all species), making a more detailed analysis unfeasible.

Despite the large number of covariates examined during our analysis, very few were useful to predict snakebite risk along the Terai. It is possible that confounders or other difficult-to-measure covariates could better explain the complex relationship between the ecology and biology of MIVS, socio-economic factors, human behavioural traits, and the circumstances around domestic animal keeping. This needs to be further explored, following a recent call for an overarching One Health and Ecohealth approach to better understand the drivers for snakebite risk, incidence, and mortality under specific situations. ${ }^{32}$

In conclusion, snakebite is a multi-factorial disease and there is no possible universal approach to model its risk. Each model should be individually designed for each set of socio-economical, geographic, ecological, cultural, and environmental circumstances. ${ }^{19}$ To better understand and address the snakebite problem, it is necessary to approach it, whenever possible, with local data collected at a national scale, so that the conclusions drawn can fuel appropriate national public health policies and actions. As long as people work, live, and keep their domestic animals in close contact with natural environments with MIVS, the risk of snakebite will be present. However, better understanding of the factors influencing that risk at the most granular scale possible, and the estimation of the populations at risk, can help to better target prevention and mitigation measures. For humans, this evidence can channel efforts towards improved access to treatment through the optimized stockpiling of antivenom, and the improvement, relocation or 
new construction of treating facilities, but more importantly, towards community education and sensitization in preventive campaigns. ${ }^{46}$ For domestic animals, this information could help better target awareness-raising activities for animal owners and implement mitigation strategies. For animals at higher risk, tailored interventions such as the improvement of housing conditions, regular cleaning of sheds and surrounding areas (e.g., from food waste and surrounding vegetation), and using light when animals are walked out of the enclosure at night could be deployed specifically as snakebite prevention measures. ${ }^{47}$ At stake is the reduction of snakebite envenoming incidence rates in humans and animals, and of its possible long-term sequelae on human populations.

\section{Methods}

Area and primary data description

The geographic focus of this study was the Terai region of Nepal, populated by about $16,087,000$ people (about $57.3 \%$ of Nepal's population) for 2018 according to the UN-adjusted estimates from WorldPop ${ }^{48}$. In Nepal, just under $80 \%$ of the population lives in rural areas ${ }^{49}$ and the Terai concentrates most of these rural population. ${ }^{9}$ Since snakebite affects primarily rural population, ${ }^{30,50,51}$ we started by filtering out urban and densely populated clusters areas where snakebite is considered to be unusual. ${ }^{21}$ The primary cluster areas used for the study were the Village Development Committees (VDC), which represented the smallest, viable administrative unit recognized at the time in Nepal. Our primary data source was a multicluster random survey, including more than 13,800 households in all the Terai. The survey was carried out between December 2018 and May 2019, and gathered retrospectively a wide range of information in addition to the snakebite cases in humans and animals during the previous 12 months. All answers were associated to the households' geolocation. The detailed survey methodology can be found in Alcoba et al. 2021. ${ }^{26}$ Two binary response variables, one for humans, and one for domestic animals, identified snakebite cases in the last 12 months (from the survey's date) and defined the two main models (human and animal). Snakebite risk is defined as the theoretical probability of encountering and being bitten by a snake. ${ }^{46}$ We considered this probability within a period of 12 months, either for a household member in the estimation model, or for anyone in a specific area of $1 \mathrm{~km}^{2}$ in the geospatial prediction model. In addition, snakebite risk can be measured as the likelihood of exposure to a snake (presence and abundance) times the likelihood of an encounter leading to a bite. ${ }^{46}$ Considering the impossibility of measuring snake abundance at national scale, in this study we regressed the incidence of snakebite on a number of additional factors to find that theoretical probability. All data management and curation, as well as the statistical analysis were done in R version 4.0.4. ${ }^{52}$ Some input and output geospatial layers were processed in QGIS version 3.16.4. ${ }^{53}$

Model architecture

The Integrated Nested Laplace Approximations (INLA) method is a computationally efficient approach for Bayesian statistical inference of latent Gaussian Markov Random Field (GMRF) models. ${ }^{54}$ These are 
highly adaptable type of models including linear, generalized, mixed, spatial, and spatio-temporal models. ${ }^{19,55}$ This type of Bayesian Hierarchical models is especially robust for the analysis of highly sparse data, such as the one presented by neglected tropical diseases like snakebite, and its integration with the Stochastic Partial Differential Equations (SPDE) ${ }^{56}$ allows to model multiple types of georeferenced data, ${ }^{19}$ while borrowing strength across space and time. ${ }^{57}$

The models for the risk of snakebite (SB) at location $i$ satisfy the following general structure:

$$
\begin{gathered}
S B\left(s_{i}\right) \sim \operatorname{Bernoulli}\left(\pi\left(s_{i}\right)\right) \\
\operatorname{logit}\left(\pi\left(s_{i}\right)\right)=\eta\left(s_{i}\right)=\beta_{0}+\mathbf{X}\left(\mathbf{s}_{i}\right) \boldsymbol{\beta}_{j}+u\left(s_{i}\right) \\
u\left(s_{i}\right) \sim \operatorname{GMRF}(\mathbf{0}, \mathbf{\Sigma})
\end{gathered}
$$

Where, $\beta_{0}$ is the intercept, $X\left(s_{i}\right) \beta_{j}$ are the covariates' matrix and coefficients, $i$ represents the surveyed households and their respective locations, $j$ is the number of covariates, $\pi\left(s_{i}\right)$ is the expected value for the risk of snakebite at location $i$, and $u\left(s_{i}\right)$ is the spatially correlated random effect. This $u$ component follows a GMRF distribution with mean zero $(0)$ and covariance matrix $\boldsymbol{\Sigma}$, which models the data's spatial dependency. ${ }^{18}$ To determine $u$, it is necessary to estimate $\boldsymbol{\Sigma}$, whose elements are defined by a Matérn covariance function (here simplified for two dimensions):

$$
\operatorname{Cov}\left(U\left(s_{i}\right), U\left(s_{i}{ }^{\prime}\right)\right)=\sigma^{2} \times\left(\kappa \times\left\|s_{i}-s_{i}{ }^{\prime}\right\|\right) \times\{\backslash \mathrm{rm} \mathrm{K}\}_{1}\left(\kappa \times\left\|s_{i}-s_{i^{\prime}}\right\|\right) \#(2)
$$

Where, $s_{i}$ and $s_{i}$, are spatial locations of the observations $i$ and $i,\|\cdot\|$ represents the Euclidean distance between two points, $\mathrm{K}$ is a scale hyperparameter and $\mathrm{K}_{1}$ is the modified Bessel function of the second kind with order 1 . For a general description of the INLA statistical approach, see the supplementary Document S1, and for a detailed description see Rue et al. 2009. ${ }^{54}$ The INLA method is implemented in the R package INLA (version 21.02.23). ${ }^{58}$

Selection of geospatial covariates

Two data sources were available for our analysis. The first was our national multi-cluster random survey, which provided highly granular observations for demographic, clinical, socio-economic and environmental covariates (Table 4). The second was a set of publicly available national gridded data sets (Table 4). Depending on the type of model (human or animal), we considered suitable covariates from the survey, and open-source geospatial gridded layers that could be relevant for the model response. These gridded layers were selected based on their possible relevance to snake ecology or behaviour as reported by previous studies with similar research focuses, analytical methodology, or environmental and ecological study conditions. Priority was given to studies addressing snakebite risk and the distribution of venomous snake species, often taken as a risk proxy. ${ }^{13-15,17,38,39}$ Most of these studies based their estimations mainly on climatic covariates from the WorldClim database, ${ }^{59}$ and on environmental covariates such as NDVI and human footprint ${ }^{14}$. However, since snakebite has been largely associated 
with poverty, ${ }^{30}$ other covariates used to address poverty related topics, for instance illiteracy, malnutrition and filariasis, ${ }^{18,19,60}$ were also considered.

Table 4

Geospatial covariates used for the current estimation of snakebite risk in humans or animals (gridded and survey based).

\begin{tabular}{|c|c|c|c|c|}
\hline Category & Covariate & Description & Scaling & Data source \\
\hline \multirow[t]{4}{*}{ Environmental } & NDVI annual average for 2018 & Continuous & No & NEO team \\
\hline & Distance to water (Euclidean distances) & Continuous & .0 .001 & $\begin{array}{l}\text { Based on } \\
\text { GeoFabrik }\end{array}$ \\
\hline & & & & and $\mathrm{OSM}^{63}$ \\
\hline & $\begin{array}{l}\text { Human modification of terrestrial } \\
\text { systems (HMTS) } 2016\end{array}$ & Continuous & No & $\begin{array}{l}\text { NASA } \\
\text { SEDAC }\end{array}$ \\
\hline Climatic & $\begin{array}{l}\text { BIO6 (min. temperature of coldest } \\
\text { month) } 1970-2000\end{array}$ & Continuous & $\cdot 0.1$ & WorldClim 59 \\
\hline \multirow[t]{3}{*}{ Epidemiological } & Food storage & $\begin{array}{l}\text { Discrete, } 2 \\
\text { levels }\end{array}$ & No & Survey \\
\hline & Straw storage & $\begin{array}{l}\text { Discrete, } 2 \\
\text { levels }\end{array}$ & No & Survey \\
\hline & Sleep on floor & $\begin{array}{l}\text { Discrete, } 2 \\
\text { levels }\end{array}$ & No & Survey \\
\hline $\begin{array}{l}\text { Socio- } \\
\text { economical }\end{array}$ & PPI & Continuous & $\cdot 0.01$ & Survey \\
\hline \multirow[t]{2}{*}{$\begin{array}{l}\text { Livestock } \\
\text { density }\end{array}$} & Pig density, 2010 & Continuous & .0 .001 & $\begin{array}{l}\text { Harvard } \\
\text { dataverse }\end{array}$ \\
\hline & Sheep density, 2010 & Continuous & .0 .001 & $\begin{array}{l}\text { Harvard } \\
\text { dataverse } \\
\text { da }^{29}\end{array}$ \\
\hline
\end{tabular}

The model selection process started with a set of 66 preliminary covariates (including available geospatial layers and relevant survey covariates), and finished with six for both the human and animal models. Highly correlated covariates were removed, first by computing the Pearson's correlation coefficients between covariate's pairs, setting a threshold of \pm 0.6 , and second by running a sequential Variance Inflation Factor (VIF) analysis with a threshold of five, also aimed at reducing multicollinearity between covariates. For each model, the selection process started with 15 to 18 covariates, which served as a baseline reference. During model selection, and after each iteration, we identified and removed parameters with posterior distribution means with negligible overall effects on the linear predictor. For that, we set a threshold between 0.1 and 0.1 (log Odds scale), which represents an effect change of just above $10 \%$. Additionally, we used the Watanabe-Akaike Information Criterion (WAIC) value ${ }^{61}$ to evaluate the goodness of fit and model iteration comparison. These two procedures helped to filter out non- 
significant covariates either with very small or mild effects and very large standard deviations. Similarly, we assessed the effect of covariate's interactions for several meaningful pairs of covariates in each model. Finally, we evaluated the importance of the spatial random effect, by also running each main model without that component.

Input preparation

To avoid numerical inconsistencies due to large-scale differences between covariates, we scaled-down in ten-folds some of the continuous covariates (see Table 4). Scaled inputs tend to work better with the default priors and to improve the performance of the models. ${ }^{62}$ Ten of the socio-economic survey covariates were transformed into the Poverty Probability Index (PPI, https://www.povertyindex.org/), as intended by design. The specific questions generating this data for Nepal can be found in the supplementary Document S1. This standardized index denotes the likelihood of a household being below a certain poverty line. In this case, we used the PPI ' $100 \%$ national' lookup table for Nepal as reference to define the poverty likelihood. ${ }^{27}$ Finally, the covariate 'Distance to water' was created by calculating Euclidean distances from each household to the nearest permanent waterbody available in OpenStreetMaps layers. ${ }^{63}$

Validation

Due to the data sparseness, validation schemes such as splitting the data in training and testing subsets could not be carried out. For the risk analysis in humans, only $1.18 \%$ of the binary responses were positive, as were $0.7 \%$ in animals. Such small rates would complicate the possibility of obtaining a representative random sample, remove any power from the tests and difficult their convergence. Instead, we performed a sensitivity analysis by constantly challenging the stability of the parameters and hyperparameters under different circumstances, until no further improvement was possible.

Geospatial risk prediction

Since the household survey covariates cannot be imputed to other locations in the Terai, for prediction purposes we implemented a variation of the human risk model. It included exclusively geospatial data available for the whole Terai region in the form of gridded data. The input data included both the survey observation points, and a regular grid of 39,684 points spaced every $1 \mathrm{~km}$, covering all the Terai, where the response variables were predicted. For each of these points, a posterior distribution was approximated and the mean and standard deviation (SD) were estimated. Finally, using the mean risk and the household population density, we mapped the estimated number of households at risk of suffering a snakebite per $\mathrm{km}^{2}$ in one year. For the animal model, it was not possible to plot a snakebite risk map, since data scarcity did not allow for the spatial analysis of individual species, and a unified risk map for more than 10 species would not convey any useful information.

\section{Declarations}


We are very grateful to Mr Mamit Rai for his in invaluable technical and logistic support during multiple stages of this research process. Many thanks to the Help Desk team at R-INLA.org (Dr Håvard Rue and Dr Finn Lindgren) for their great responsiveness answering multiple technical questions. Dr Haakon Bakka gave valuable suggestions to improve our statistical models. We thank Dr Andrew M. Durso for his valuable opinions on selection and interpretation of environmental covariates. We also thank the surveyors, the local leaders and the surveyed population for their essential cooperation.

\section{Funding}

The Swiss National Science Foundation (SNSF) funded this study under the project number 315130_176271. Gabriel Alcoba was partly funded by MSF (Médecins sans Frontières/Doctors without Borders). Rafael Ruiz de Castañeda was partly funded by Fondation Louis Jeantet.

Role of the funding source

The sponsors of the study had no role in study design, data collection, data analysis, data interpretation, or writing of the manuscript.

Data availability

The data used and the sources are described in this article and in the supplementary materials. For the primary data collected in the Snake-Byte survey, participant data that underlie the results reported in this article will be made available upon reasonable request through the University of Geneva data repository, after de-identification, beginning 12 months following the publication of this article. Requests should be directed to nicolas.ray@unige.ch

Code availability

The base code for reproducing the INLA analysis is available in the supplementary R-script S1.

Author contributions

CO designed the spatial sampling methodology, conceptualized the analysis, analyzed and interpreted the data, produced the output figures, and wrote the initial manuscript. CO and MP conducted the statistical analysis, including the model selection and interpretation. NR, FC, RRdC, IB, GA, and SKS conceived and designed the study. FC and NR oversaw the Snake-Byte project and obtained the funding. CO, SBM, IB, GA, SKS, RRdC, NR, and FC were involved in the survey design. SKS directed the field data collection. All authors contributed to subsequent revisions and approved the final version submitted for publication. NR, FC and $\mathrm{CO}$ had the final responsibility for the decision to submit for publication. 
The authors declare no competing interests.

\section{References}

1 Bawaskar, H. S. Snake bite poisoning: a neglected life-threatening occupational hazard. Indian J Crit Care Med18, 123-124, doi:10.4103/0972-5229.128698 (2014).

2 Gutiérrez, J. M. et al. Snakebite envenoming. Nature Reviews Disease Primers3, 1-21 (2017).

3 Williams, D. J. et al. Strategy for a globally coordinated response to a priority neglected tropical disease: Snakebite envenoming. PLoS neglected tropical diseases13, e0007059, doi:10.1371/journal.pntd.0007059 (2019).

4 WHO. Snakebite envenoming: a strategy for prevention and control. (2019).

5 Warrell, D. A. Snake bite. The Lancet375, 77-88 (2010).

6 Bolon, I. et al. Snakebite in domestic animals: first global scoping review. Preventive veterinary medicine170, 104729 (2019).

7 Babo Martins, S. et al. Snakebite and its impact in rural communities: The need for a One Health approach. PLoS neglected tropical diseases13, e0007608, doi:10.1371/journal.pntd.0007608 (2019).

8 LIVESTOCK STATISTICS OF NEPAL 2016/17 (2073/74). (Ministry of Agriculture, Land Management and Cooperatives, Hariharbhawan, Lalitpur, Nepal, 2018).

9 STATISTICAL YEAR BOOK OF NEPAL - 2017. (Central Bureau of Statistics, Ramshahpath, Thapathali, Kathmandu, Nepal, 2018).

10 Chaudhary, S., Singh, S., Chaudhary, N. \& Mahato, S. Snake-bite in Nepal. Journal of Universal College of Medical Sciences2, 45-53 (2014).

11 Alcoba, G. et al. Snakebite epidemiology in humans and domestic animals across Nepal's Terai: a multi-cluster random survey. Preprint at http://dx.doi.org/10.2139/ssrn.3867686 (2021).

12 Bolon, I. et al. What is the impact of snakebite envenoming on domestic animals? A nation-wide community-based study in Nepal and Cameroon. Toxicon: $X, 100068$, doi:https://doi.org/10.1016/j.toxcx.2021.100068 (2021).

13 Zacarias, D. \& Loyola, R. Climate change impacts on the distribution of venomous snakes and snakebite risk in Mozambique. Climatic Change152, 195-207 (2019).

14 Yousefi, M., Kafash, A., Khani, A. \& Nabati, N. Applying species distribution models in public health research by predicting snakebite risk using venomous snakes' habitat suitability as an indicating factor. Scientific reports10, 1-11 (2020). 
15 Yañez-Arenas, C., Díaz-Gamboa, L., Patrón-Rivero, C., López-Reyes, K. \& Chiappa-Carrara, X. Estimating geographic patterns of ophidism risk in Ecuador. Neotropical Biodiversity4, 55-61 (2018).

16 Longbottom, J. et al. Vulnerability to snakebite envenoming: a global mapping of hotspots. The Lancet392, 673-684, doi:10.1016/S0140-6736(18)31224-8 (2018).

17 Yañez-Arenas, C., Peterson, A. T., Rodríguez-Medina, K. \& Barve, N. Mapping current and future potential snakebite risk in the new world. Climatic change134, 697-711 (2016).

18 Utazi, C. E. et al. High resolution age-structured mapping of childhood vaccination coverage in low and middle income countries. Vaccine36, 1583-1591 (2018).

19 Bosco, C. et al. Exploring the high-resolution mapping of gender-disaggregated development indicators. Journal of The Royal Society Interface14, 20160825 (2017).

20 Rahman, R. et al. Annual incidence of snake bite in rural Bangladesh. PLoS Neglected Tropical Diseases4, e860 (2010).

21 Ediriweera, D. S. et al. Mapping the risk of snakebite in Sri Lanka - a national survey with geospatial analysis. PLoS Neglected Tropical Diseases10, e0004813, doi:10.1371/journal.pntd.0004813 (2016).

22 Sharma, S. K. et al. Impact of snake bites and determinants of fatal outcomes in southeastern nepal. The American Society of Tropical Medicine and Hygiene71, 234-238 (2004).

23 Magar, C. T. et al. A hospital based epidemiological study of snakebite in Western Development Region, Nepal. Toxicon69, 98-102 (2013).

24 Sharma, S. K., Khanalb, B., Pokhrelc, P., Khana, A. \& Koirala, S. Snakebite-reappraisal of the situation in Eastern Nepal. Toxicon41, 285-289 (2003).

25 in WHO NTD department 28 (World Health Organization, Geneva, 2017).

26 Alcoba, G. et al. Novel transdisciplinary methodology for cross-sectional analysis of snakebite epidemiology at national scale. PLOS Neglected Tropical Diseases15, e0009023, doi:https://doi.org/10.1371/journal.pntd.0009023 (2021).

27 Schreiner, M. PPI scorecard for Nepal, <https://www.povertyindex.org/country/nepal> (2010).

28 Kennedy, C. M., Oakleaf, J. R., Theobald, D. M., Baruch-Mordo, S. \& Kiesecker, J. Managing the middle: a shift in conservation priorities based on the global human modification gradient. Global Change Biology25, 811-826 (2019).

29 Gilbert, M. et al. Global distribution data for cattle, buffaloes, horses, sheep, goats, pigs, chickens and ducks in 2010. Scientific data5, 1-11 (2018). 
30 Harrison, R. A., Hargreaves, A., Wagstaff, S. C., Faragher, B. \& Lalloo, D. G. Snake envenoming: a disease of poverty. PLoS Neglected Tropical Diseases3, e569, doi:10.1371/journal.pntd.0000569 (2009).

31 Chaves, L. F., Chuang, T.-W., Sasa, M. \& Gutiérrez, J. M. Snakebites are associated with poverty, weather fluctuations, and El Niño. Science advances1, e1500249 (2015).

32 Gutiérrez, J. M. Snakebite envenoming from an Ecohealth perspective. Toxicon: X7, 100043 (2020).

33 Bawaskar, H. \& Bawaskar, P. Profile of snakebite envenoming in western Maharashtra, India.

Transactions of the Royal Society of Tropical Medicine and Hygiene96, 79-84 (2002).

34 Saluba Bawaskar, H. \& Himmatrao Bawaskar, P. Envenoming by the common krait (Bungarus caeruleus) and Asian cobra (Naja naja): clinical manifestations and their management in a rural setting. Wilderness \& Environmental Medicine15, 257-266, doi:https://doi.org/10.1580/10806032(2004)015[0257:EBTCKB]2.0.C0;2 (2004).

35 Chappuis, F., Sharma, S. K., Jha, N., Loutan, L. \& Bovier, P. A. Protection against snake bites by sleeping under a bed net in southeastern Nepal. The American journal of tropical medicine and hygiene77, 197199 (2007).

36 Southwood, A. \& Avens, L. Physiological, behavioral, and ecological aspects of migration in reptiles. Journal of Comparative Physiology B180, 1-23, doi:10.1007/s00360-009-0415-8 (2010).

37 WHO. Guidelines for the management of snake-bites. (World Health Organization, 2010).

38 Molesworth, A. M., Harrison, R., David, R., Theakston, G. \& Lalloo, D. G. Geographic Information System mapping of snakebite incidence in northern Ghana and Nigeria using environmental indicators: a preliminary study. Transactions of the Royal Society of Tropical Medicine and Hygiene97, 188-192 (2003).

39 Schneider, M. C. et al. Overview of snakebite in Brazil: possible drivers and a tool for risk mapping. PLoS neglected tropical diseases15, e0009044 (2021).

40 Macartney, J. M., Larsen, K. W. \& Gregory, P. T. Body temperatures and movements of hibernating snakes (Crotalus and Thamnophis) and thermal gradients of natural hibernacula. Canadian Journal of Zoology67, 108-114 (1989).

41 Sperry, J. H. \& Weatherhead, P. J. Prey-mediated effects of drought on condition and survival of a terrestrial snake. Ecology89, 2770-2776 (2008).

42 Brown, G. P. \& Shine, R. Rain, prey and predators: climatically driven shifts in frog abundance modify reproductive allometry in a tropical snake. Oecologia154, 361-368 (2007). 
43 Hansson, E., Sasa, M., Mattisson, K., Robles, A. \& Gutierrez, J. M. Using geographical information systems to identify populations in need of improved accessibility to antivenom treatment for snakebite envenoming in Costa Rica. PLoS Negl Trop Dis7, e2009, doi:10.1371/journal.pntd.0002009 (2013).

44 Roll, U. et al. The global distribution of tetrapods reveals a need for targeted reptile conservation. Nature Ecology \& Evolution1, 1677-1682 (2017).

45 Luiselli, L., Sale, L., Akani, G. C. \& Amori, G. Venomous snake abundance within snake species' assemblages Worldwide. Diversity12, 69 (2020).

46 Pintor, A. F. et al. Addressing the global snakebite crisis with geo-spatial analyses-Recent advances and future direction. Toxicon: $X, 100076$ (2021).

47 Joshi, V., Alam, S. \& Dimri, U. Snake bites in farm animals: a field guide. Indian Dairyman (2018). <https://www.researchgate.net/publication/323225477_Snake_Bites_in_Farm_Animals_A_Field_Guide>.

48 WorldPop. Global high resolution population denominators project funded by the Bill and Melinda Gates Foundation (OPP1134076). School of Geography and Environmental Science, University of Southampton; Department of Geography and Geosciences, University of Louisville; Departement de Geographie, Universite de Namur, and Center for International Earth Science Information Network (CIESIN), Columbia University (2018), doi:https://dx.doi.org/10.5258/SOTON/WP00675 (2020).

49 United Nations Department of Economic and social Affairs, P. D. World urbanization prospects: the 2018 revision, <https://population.un.org/wup/Country-Profiles/> (2018).

50 Kasturiratne, A. et al. The global burden of snakebite: a literature analysis and modelling based on regional estimates of envenoming and deaths. PLoS Med5, e218, doi:10.1371/journal.pmed.0050218 (2008).

51 Chippaux, J. P. Estimate of the burden of snakebites in sub-Saharan Africa: a meta-analytic approach. Toxicon57, 586-599, doi:10.1016/j.toxicon.2010.12.022 (2011).

52 R: A language and environment for statistical computing (R Foundation for Statistical Computing, Vienna, Austria, 2020).

53 QGIS Geographic Information System v. 3.16.4 (QGIS Development Team, 2021).

54 Rue, H., Martino, S. \& Chopin, N. Approximate Bayesian inference for latent Gaussian models by using integrated nested Laplace approximations. Journal of the royal statistical society: Series b (statistical methodology)71, 319-392 (2009).

55 Utazi, C. E. et al. A spatial regression model for the disaggregation of areal unit based data to highresolution grids with application to vaccination coverage mapping. Statistical Methods in Medical Research28, 3226-3241 (2019). 
56 Lindgren, F., Rue, H. \& Lindström, J. An explicit link between Gaussian fields and Gaussian Markov random fields: the stochastic partial differential equation approach. Journal of the Royal Statistical Society: Series B (Statistical Methodology)73, 423-498 (2011).

57 Moraga, P. Geospatial Health Data: Modeling and Visualization with R-INLA and Shiny. (CRC Press, 2019).

58 Lindgren, F. \& Rue, H. Bayesian spatial modelling with R-INLA. Journal of Statistical Software63, 1-25 (2015).

59 Fick, S. E. \& Hijmans, R. J. WorldClim 2: new 1-km spatial resolution climate surfaces for global land areas. International journal of climatology37, 4302-4315 (2017).

60 Moraga, P. et al. Modelling the distribution and transmission intensity of lymphatic filariasis in subSaharan Africa prior to scaling up interventions: integrated use of geostatistical and mathematical modelling. Parasites \& vectors8, 1-16 (2015).

61 Watanabe, S. \& Opper, M. Asymptotic equivalence of Bayes cross validation and widely applicable information criterion in singular learning theory. Journal of machine learning research11 (2010).

62 Zuur, A. F., leno, E. N. \& Saveliev, A. A. Beginner's Guide to Spatial, Temporal, and Spatial-Temporal Ecological Data Analysis with R-INLA. Vol. 1 (Highland Statistics Ltd, 2017).

63 Ramm, F. et al. OpenStreetMap data in layered GIS format. Version 0.67 (2014).

\section{Figures}




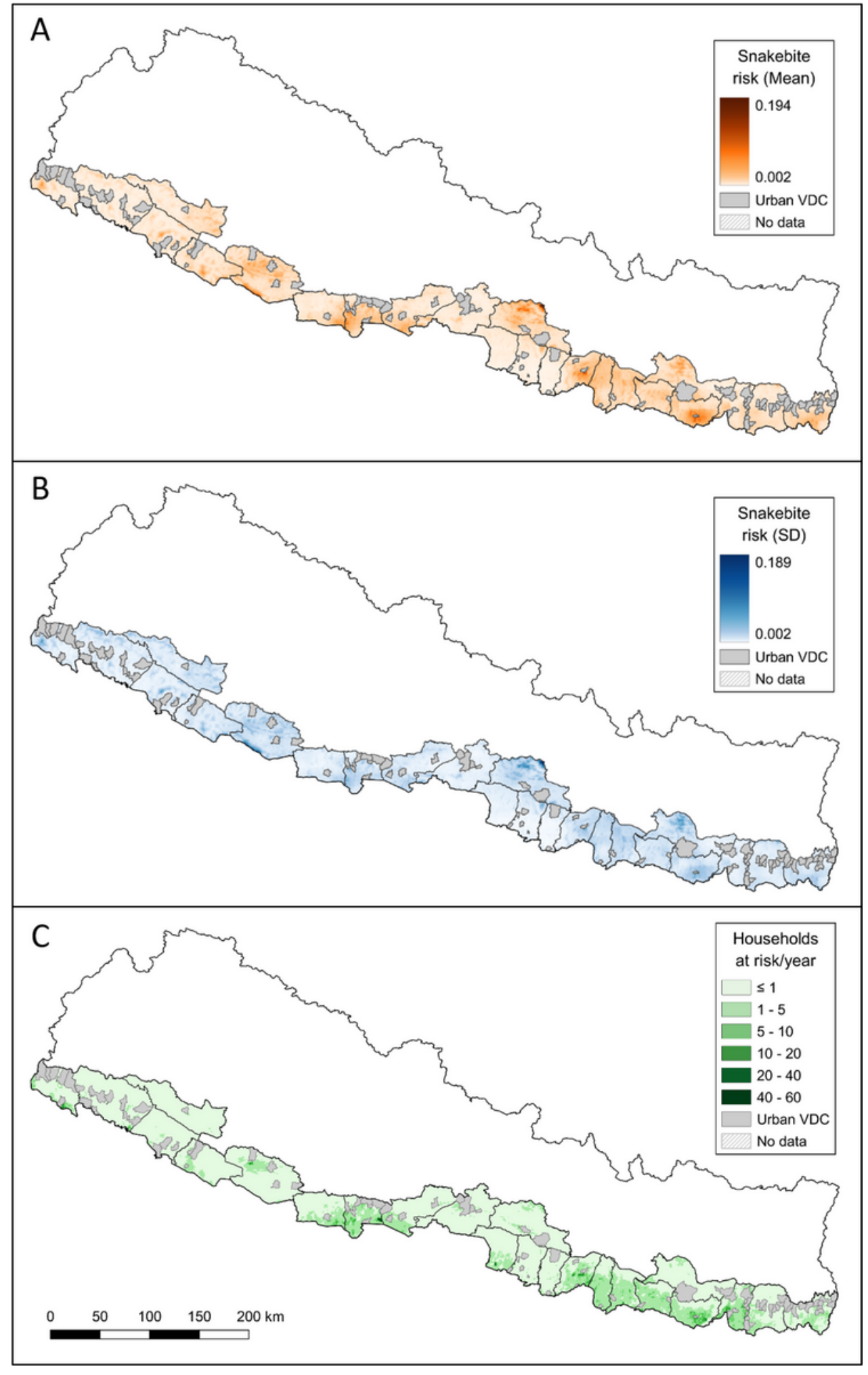

\section{Figure 1}

(A) Mean posterior distribution, (B) uncertainty (SD) of the snakebite risk for the Terai at $1 \mathrm{~km} 2$ resolution, and (C) estimated number of households at risk of snakebite per $1 \mathrm{~km} 2 /$ year, based on the WorldPop UNadjusted population estimates for Nepal in 2018. 


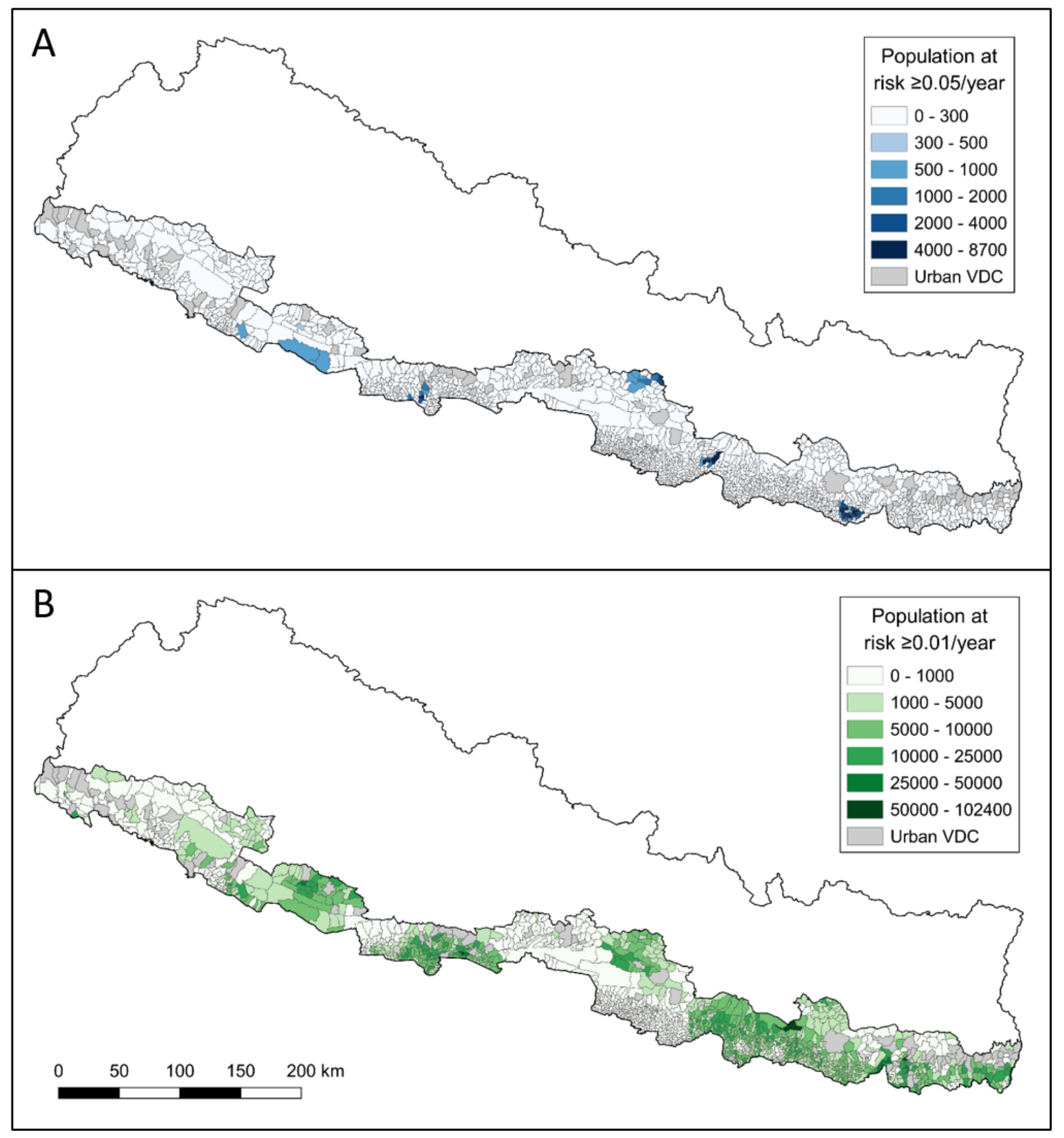

Figure 2

Choropleth maps aggregating the estimated population (WorldPop UN-adjusted for 2018) exposed at snakebite risks $\geq 0.05(A)$ and $(B) \geq 0.01$ per Village Development Committee during 12 months.

\section{Supplementary Files}


This is a list of supplementary files associated with this preprint. Click to download.

- S1Supplementarydocument.pdf

- S1Rscript.txt

- S2PopatriskMunicipality0.05.html

- S3PopatriskDistrict0.05.html

- S4PopatriskVDC0.01.html

- S5PopatriskMunicipality0.01.html

- S6PopatriskDistrict0.01.html 